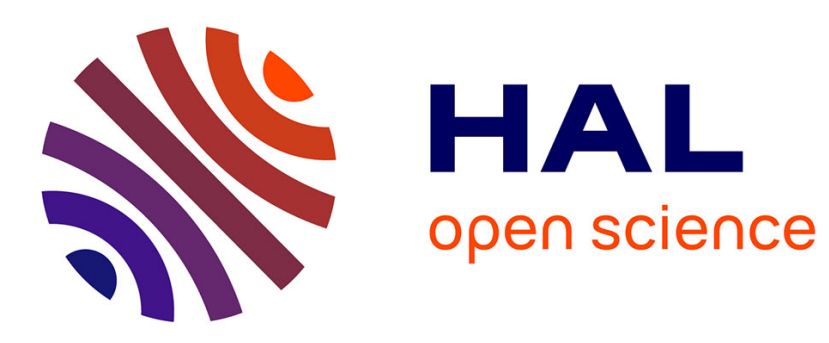

\title{
Energy stability analysis for a hybrid fluid-kinetic plasma model
}

Philip Morrison, Emanuele Tassi, Cesare Tronci

\section{To cite this version:}

Philip Morrison, Emanuele Tassi, Cesare Tronci. Energy stability analysis for a hybrid fluid-kinetic plasma model. Nonlinear Physical Systems: Spectral Analysis, Stability and Bifurcations, Nov 2012, Banff, Canada. hal-01053857

\section{HAL Id: hal-01053857 https://hal.science/hal-01053857}

Submitted on 2 Aug 2014

HAL is a multi-disciplinary open access archive for the deposit and dissemination of scientific research documents, whether they are published or not. The documents may come from teaching and research institutions in France or abroad, or from public or private research centers.
L'archive ouverte pluridisciplinaire HAL, est destinée au dépôt et à la diffusion de documents scientifiques de niveau recherche, publiés ou non, émanant des établissements d'enseignement et de recherche français ou étrangers, des laboratoires publics ou privés. 


\section{Chapter 14}

\section{Energy Stability Analysis for a Hybrid Fluid-Kinetic Plasma Model}

In plasma physics, a hybrid fluid-kinetic model is composed of a magnetohydrodynamics (MHD) part that describes a bulk fluid component and a Vlasov kinetic theory part that describes an energetic plasma component. Although most hybrid models in the plasma literature are non-Hamiltonian, this chapter investigates a recent Hamiltonian variant in its two-dimensional configuration. The corresponding Hamiltonian structure is described along with its Casimir invariants. Then, the energy-Casimir method is used to derive explicit sufficient stability conditions, which imply a stable spectrum and suggest nonlinear stability.

\subsection{Introduction}

The discipline of plasma physics has provided a rich collection of spectral and stability problems. Depending on the circumstances, plasmas may be described by fluid models or kinetic theories with coupling to electromagnetic fields. As a result, plasma theory has all of the possibilities and concomitant complications of all these disciplines and, although a great deal of lore has been generated on the formal level, there remain many open mathematical problems of a spectral and stability nature.

When dissipative terms are small enough to be safely neglected, the resulting plasma systems are infinite-dimensional Hamiltonian systems. Thus, we are led to stability and spectral problems of a wide variety of Hamiltonian operators. Usually, these operators are non-Hermitian, non-normal and have rich spectra with both point

Chapter written by Philip J. MORRISON, Emanuele TASSI and Cesare TrONCI. 
and continuous components. Bifurcation theory of these operators including their nonlinear extensions provides challenging non-trivial problems of important physical relevance.

A particularly challenging class of spectral and stability problems arises from the so-called hybrid models of plasma physics. These are models that incorporate both fluid and kinetic equations. Generally speaking, the purpose of these models is to describe a bulk portion of the plasma by a fluid model, such as MHD, while describing a hot component of the plasma by a kinetic theory, such as the Maxwell-Vlasov system. Thus, hybrid models can combine all spectral and stability issues that occur in fluid and kinetic theories separately into a complicated whole. It is well known that MHD and the Vlasov equation separately have a variety of interesting point and continuous components to their spectra, and so, hybrid models can indeed present a challenging class of mathematical problems. However, because of the Hamiltonian nature of these models, we can use energy techniques to obtain spectral information without performing detailed operator analysis. In particular, we can obtain sufficient conditions for the existence of a stable spectrum by the so-called energy-Casimir method (see [HOL 85, MOR 98]) that is based on the natural Lyapunov-Dirichlet method of Hamiltonian systems theory.

The purpose of this chapter is to describe a particular hybrid model that is a coupling between two-dimensional (planar) MHD and Vlasov theory. We will describe its Hamiltonian structure and apply the energy-Casimir method to a class of equilibrium states and obtain sufficient conditions for stability. This chapter is organized as follows. In section 14.2, we review some details regarding stability and the energy-Casimir method. Then, in section 14.3, we describe the planar hybrid model, its noncanonical Hamiltonian structure and associated Casimir invariants. This is followed in section 14.4 by the application of the energy-Casimir method giving rise to the sufficient conditions. Finally, we conclude in section 14.5. For completeness, two appendices are included: Appendix A (section 14.7) provides a proof of the Jacobi identity, while Appendix B (section 14.8) contains a direct verification that the functional indicated as Casimir for the hybrid model, indeed, commutes with every other observable.

\subsection{Stability and the energy-Casimir method}

Consider a dynamical system $\dot{z}=V$ defined on some space (manifold) $\mathscr{Z}$, where $z \in \mathscr{Z}$ can be a point or a trajectory, " " " denotes time derivative and $V$ is an autonomous vector field defined on $\mathscr{Z}$. Our interest is in the stability of equilibrium points $z_{e}$, solutions that satisfy $V\left(z_{e}\right)=0$. We adopt the standard definition of stability for such points, which is the following:

An equilibrium point $z_{e}$, of a dynamical system is said to be stable if, for any neighborhood $N$ of $z_{e}$ there exists a subneighborhood $S \subset N$ of 
$z_{e}$, such that if $\stackrel{\circ}{z}$, an initial condition, is in $S$, then the trajectory $z(t) \in N$ for all time $t>0$.

Often, we consider the associated linear problem $\delta \dot{z}=D V\left(z_{e}\right) \cdot \delta z$ obtained by expanding $V\left(z_{e}+\delta z\right)$ to first order. If $\delta z$ remains in $N$, then the system is said to be linearly stable, and to distinguish this kind of stability from that with dynamics under the full vector field $V$, we add the adjective nonlinear to describe the latter. Assuming a solution of the form $\delta z=\hat{z} \exp (\lambda t)$, the linear problem becomes $(D V-\lambda$ id $) \cdot \hat{z}=0$, where id is the identity operator. The spectrum of $D V, \sigma(D V)$, is the set composed of $\lambda \in \mathbb{C}$ for which the linear operator $D V-\lambda$ id has no densely defined continuous inverse, and an equilibrium point is said to be spectrally stable if $i \sigma(D V) \subset \overline{\mathrm{LHP}}$ (closure of the lower half complex plane). Observe that this definition of stability includes the case $i \lambda \in \mathbb{R}$, which corresponds to pure oscillation, a case that is sometimes called neutral stability. Inclusion of this case is most important, since this is the only kind of spectral stability possessed by Hamiltonian systems.

There are several logical implications between the different types of stability, and these can be somewhat subtle: for example, linear stability implies spectral stability; linear stability does not imply nonlinear stability; nonlinear stability does not imply linear stability. For establishing linear stability in Hamiltonian systems, which would assure us that the spectrum lines on $i \mathbb{R}$, we can use Lyapunov function techniques dating back to Lagrange and Dirichlet. Our main concern in this chapter is to do this for a particular hybrid model.

For Hamiltonian systems, the vector field of our dynamical system is generated by a Poisson bracket so that the equations of motion have the form $\dot{z}=\{z, H\}$, which for finite-dimensional systems in a coordinate patch is given by $\dot{z}^{i}=J^{i j} \partial_{j} H$, where $J$ is the Poisson bivector (cosymplectic form) and $\{\}:, C^{\infty}(\mathscr{Z}) \times C^{\infty}(\mathscr{Z}) \rightarrow C^{\infty}(\mathscr{Z})$. Thus, equilibrium points satisfy $d H \in \operatorname{Ker}(J)$. A consequence of the Poisson bracket identities (see Appendix A (section 14.7)) is the Lie-Darboux theorem, which implies $\operatorname{Ker}(J)$ is spanned by Casimir invariants, which satisfy $\{C, f\}=0$, for all functions $f \in C^{\infty}(\mathscr{Z})$ (although there are serious unresolved issues with this theorem for infinite-dimensional systems (see, e.g., [YOS 13])). Thus, equilibria are critical points of an invariant energy function $\mathscr{F}=H+C$ and this fact is very useful for establishing stability criteria.

For finite-dimensional systems, definiteness (positive or negative) of the quadratic form $\delta^{2} \mathscr{F}\left(z_{e} ; \delta z\right)$ assures both linear and nonlinear stability. For linear systems, $\delta^{2} \mathscr{F}$ is invariant, in fact the Hamiltonian for the linear dynamics, and its definiteness means the equilibrium point is foliated by nested invariant sets that are topologically spheres. The interior of these sets can thus serve as the subneighborhoods $S$ in the above definition of stability. For nonlinear systems, $\mathscr{F}$ is invariant and, under mild smoothness conditions, $\delta^{2} \mathscr{F}$ determines the topological character of the level sets of $\mathscr{F}$ in a sufficiently small neighborhood of the equilibrium point $z_{e}$. This guarantees 
that for any neighborhood $N$, there is an $S \subset N$, determined by some level set of $\mathscr{F}$, within which the flow must remain.

For infinite-dimensional systems, the situation is considerably more complicated. First, definiteness of $\delta^{2} \mathscr{F}$ does not imply that an extremal point that satisfies $\delta \mathscr{F}=0$ is in fact an extermum (maximum or minimum). For both linear and nonlinear stability, we require $\delta^{2} \mathscr{F}$ to lead to a norm for defining open sets. This leads to a second and for nonlinear stability an often formidable complication: even if a norm can be extracted from $\delta^{2} \mathscr{F}$ for a rigorous proof of nonlinear stability, we must show that the solution to the dynamical system actually exists in this norm. Unfortunately, for the systems of most physical significance in plasma physics, global existence results are not available. In the rosy situation where existence results are firm, it can turn out that more than one choice of norm may be available and a given equilibrium can be stable in one norm and not in another; in this case, a physical determination must be made about what is important.

In this chapter, we will follow the practice in the physics literature (e.g. [HAZ 84, HOL 85, MOR 86, MOR 98]) and only obtain formal stability criteria for our hybrid models. More specifically, in section 14.4, we will find conditions under which $\delta^{2} \mathscr{F}$ is positive definite. The reader interested in seeing what makes up a rigorous application of the energy-Casimir method is referred to [BAT 95, REI 94].

\subsection{Planar Hamiltonian hybrid model}

Many hybrid models exist in the plasma physics literature, but one of the most popular kinetic-MHD variants is that of [CHE 91, PAR 92, PAR 99, FU 95], which has often been used in computer simulations [KIM 04, TAK 09]. This model employs the so-called pressure-coupling scheme, which suffers from not conserving energy exactly. Recently, a Hamiltonian version of this scheme (HPCS) was given in [HOL 12, TRO 10, TRO 13]. Here, we will present and analyze a two-dimensional variant of the HPCS. The equations of motion will be given in section 14.3.1, its Hamiltonian structure is given in section 14.3.2 and its Casimir invariants is given in section 14.3.3.

\subsubsection{Planar hybrid model equations of motion}

Upon setting all physical constants equal to unity, the planar Hamiltonian hybrid model is given by the following system of partial differential equations: 


$$
\begin{aligned}
& \partial_{t} \omega=[\psi, \omega]+[J, A]+\hat{z} \times \nabla \cdot\left(\nabla_{\perp} \cdot \int \mathrm{d}^{3} v f \mathbf{v}_{\perp} \mathbf{v}_{\perp}\right), \\
& \begin{aligned}
\partial_{t} A=[\psi, A], \\
\frac{\partial f}{\partial t}+[f, \psi]+\mathbf{v}_{\perp} \cdot \frac{\partial f}{\partial \mathbf{x}_{\perp}}+\mathbf{v} \cdot \hat{z} \times \nabla\left(\nabla_{\perp} \psi \cdot \frac{\partial f}{\partial \mathbf{v}_{\perp}}\right) \\
\\
\quad+v_{z} \nabla_{\perp} A \cdot \frac{\partial f}{\partial \mathbf{v}_{\perp}}-\mathbf{v}_{\perp} \cdot \nabla_{\perp} A \frac{\partial f}{\partial v_{z}}=0 .
\end{aligned}
\end{aligned}
$$

In these equations, the scalar functions $A$ and $\omega$ are defined on a domain $\mathscr{D} \subseteq \mathbb{R}^{2}$ and indicate the magnetic poloidal flux function and the vorticity of the bulk flow. These are related to the magnetic field $\mathbf{B}$ and to the bulk velocity field $\boldsymbol{U}$ by

$$
\omega=\hat{z} \times \nabla \cdot \mathbf{U}:=\hat{z} \cdot \nabla \times \mathbf{U}, \quad \mathbf{B}=-\hat{z} \times \nabla A,
$$

where $\hat{z}$ indicates the unit vector along the coordinate $z$ of a Cartesian system $(x, y, z)$, in which the coordinates $x$ and $y$ cover the domain $\mathscr{D}$. The two-dimensional gradient $\nabla_{\perp}$ acts as $\nabla_{\perp} u=\hat{x} \partial_{x} u+\hat{y} \partial_{y} u$ on a generic function $u$. The current density $J$ and the stream function $\psi$, on the other hand, are related to the magnetic flux function and to the vorticity by

$$
J=-\Delta A, \quad \psi=-\Delta^{-1} \omega
$$

respectively. In [14.4], the symbol $\Delta$ denotes the two-dimensional Laplacian. The distribution function (phase space density) $f\left(\mathbf{x}_{\perp}, \mathbf{v}\right)$ is defined over the (reduced) particle phase space $\mathscr{D} \times \mathbb{R}^{3}$, where $\mathbf{x}_{\perp}$ and $\mathbf{v}_{\perp}$ denote $(x, y)$ and $\left(v_{x}, v_{y}\right)$, respectively. Finally, we indicated by $[$,$] the canonical bracket acting on two functions as f$ and $g$ by $[f, g]:=\nabla_{\perp} g \cdot \hat{z} \times \nabla f$.

Equations [14.1]-[14.3] govern the evolution of an incompressible MHD bulk system, coupled with a kinetic particle population. Equation [14.1] is a vorticity equation, in which the bulk plasma flow is affected by the presence of the kinetic species, through the additional pressure divergence term, represented by the last term on the right-hand side of equation [14.1]. In the absence of such term, we retrieve the two-dimensional version of classical MHD. Analogously, equation [14.3] describes the evolution of the distribution function of the kinetic species, which, in its turn, is influenced by the transport and force term associated with the bulk velocity. Such effects vanish upon setting $\psi=0$ in [14.3]. Equation [14.2], on the other hand, is the ideal Ohm's law reflecting the assumption that the magnetic flux is frozen into the bulk fluid. 


\subsubsection{Hamiltonian structure}

The model of equations [14.1]-[14.3] is easily obtained from the three-dimensional Hamiltonian hybrid model in [TRO 10, TRO 13] by reduction to two spatial dimensions. Thus, it is not surprising that it inherits a Hamiltonian structure in terms of a noncanonical Poisson bracket. In Appendix A (section 14.7), we show how to reduce the Poisson bracket of the three-dimensional model to obtain the planar system [14.1]-[14.3].

Recall that a Poisson bracket $\{$,$\} defines a Lie algebra realization on a set of$ observables consisting of the functionals of the dynamic variables, which here are $\omega$, $A$ and $f$. As alluded to in section 14.2, time evolution of an element $F$ of such algebra is determined by the equation

$$
\partial_{t} F=\{F, H\}
$$

where, for the case at hand, the Hamiltonian $H$ is given by

$$
H=\frac{1}{2} \int \mathrm{d}^{2} x\left(-\omega \Delta^{-1} \omega-A \Delta A+\int \mathrm{d}^{3} v f|\mathbf{v}|^{2}\right)
$$

and the expression for the Poisson bracket reads

$$
\begin{aligned}
\{F, G\}= & \int \mathrm{d}^{2} x \omega\left[\frac{\delta F}{\delta \omega}, \frac{\delta G}{\delta \omega}\right]+\int \mathrm{d}^{2} x A\left(\left[\frac{\delta F}{\delta \omega}, \frac{\delta G}{\delta A}\right]-\left[\frac{\delta G}{\delta \omega}, \frac{\delta F}{\delta A}\right]\right) \\
& -\int \mathrm{d}^{2} x \mathrm{~d}^{3} v f\left(\left[\frac{\delta F}{\delta f}, \mathbf{v} \cdot \hat{z} \times \nabla \frac{\delta G}{\delta \omega}\right]_{v}-\left[\frac{\delta G}{\delta f}, \mathbf{v} \cdot \hat{z} \times \nabla \frac{\delta F}{\delta \omega}\right]_{v}\right) \\
& +\int \mathrm{d}^{2} x \mathrm{~d}^{3} v f\left(\left[\frac{\delta F}{\delta f}, \frac{\delta G}{\delta f}\right]_{v}\right. \\
& \left.+\nabla_{\perp} A \cdot\left(\frac{\partial}{\partial v_{z}} \frac{\delta G}{\delta f} \frac{\partial}{\partial \mathbf{v}_{\perp}} \frac{\delta F}{\delta f}-\frac{\partial}{\partial v_{z}} \frac{\delta F}{\delta f} \frac{\partial}{\partial \mathbf{v}_{\perp}} \frac{\delta G}{\delta f}\right)\right)
\end{aligned}
$$

This bilinear operation satisfies antisymmetry, the Leibniz identity and the Jacobi identity (see Appendix A (section 14.7)). In [14.7] we introduced a canonical bracket defined over a reduced phase space as $[f, g]_{v}:=\nabla_{\perp} f \cdot \partial_{\mathbf{v}_{\perp}} g-\nabla_{\perp} g \cdot \partial_{\mathbf{v}_{\perp}} f$.

For the choices $F=\omega, A$, or $f$, using [14.6] and [14.7] in [14.5], we retrieve the model equations [14.1]-[14.3], provided that boundary terms arising from integration by parts vanish. This is accomplished, for instance, if the involved functions are periodic on $\mathscr{D}$ or, in case $\mathscr{D}$ is unbounded, if they also decay at infinity. The functions 
depending on the velocity coordinates are also assumed to go to zero sufficiently fast as $\mathbf{v} \rightarrow \infty$.

Concerning the Hamiltonian [14.6], we remark that it naturally expresses the total energy of the system, consisting of the sum of the bulk kinetic energy, the magnetic energy and the kinetic energy of the hot particle population, corresponding to the three terms appearing in [14.6], respectively.

With regard to the Poisson bracket, on the other hand, we observe that it possesses a pure MHD part, consisting of the first two terms of [14.7], which correspond to the Poisson bracket of reduced MHD [MOR 84, MAR 84]. It also possesses a purely kinetic part, given by its last two terms, which include the Vlasov bracket [MOR 80a, MOR 82, MAR 82]. The remaining terms, on the other hand, are those responsible for the coupling between the MHD and the kinetic components.

\subsubsection{Casimir invariants}

As discussed in section 14.2, the energy-Casimir method requires the identification of the Casimir invariants $C$, i.e. functionals satisfying $\{F, C\}=0$ for any arbitrary functional $F$ in the algebra of observables. However, finding the Casimirs is not always an easy task and their limited availability sometimes stands as the major obstacle to the application of the method. However, in the case under consideration, the existence of a cross-helicity invariant for the Hamiltonian PCS was shown in [HOL 12] and this Casimir finds its way into the present two-dimensional theory, where it is generalized. The existence of such a Casimir for the three-dimensional Poisson bracket yields a whole family of Casimir invariants when projected on the plane. Upon summing contributions arising from the magnetic helicity and the Vlasov dynamics, the total Casimir invariant for the planar hybrid model reads

$$
C(\omega, A, f)=\int \mathrm{d}^{2} x\left(\bar{\omega} \Phi(A)+\Psi(A)+\int \mathrm{d}^{3} v \Lambda(f)\right)
$$

where $\Phi, \Psi$ and $\Lambda$ are arbitrary functions, and we have introduced the shorthand

$$
\bar{\omega}:=\omega-\hat{z} \times \nabla \cdot \mathbf{K}, \quad \text { with } \quad \mathbf{K}:=\int \mathrm{d}^{3} v f \mathbf{v}_{\perp}
$$

That [14.8] is in fact a Casimir invariant is shown in Appendix B (section 14.8).

The existence of such Casimir invariants is amenable to a physical interpretation, which becomes clearer when considering separately, the contributions coming from 
the functions $\Phi, \Psi$ and $\Lambda$. If $\Phi \equiv \Lambda \equiv 0$, the remaining Casimir expresses the conservation of magnetic flux through a surface moving with the bulk fluid velocity. This property is the frozen-in condition for the magnetic flux, inherited from ideal MHD. If $\Phi \equiv \Psi \equiv 0$, we retrieve the conservation of the integral of any function of $f$, which is characteristic of Vlasov systems and whose physical meaning in terms of particle rearrangements was given in [MOR 87]. However, a new family of invariants associated with this two-dimensional hybrid model appears when setting $\Psi \equiv \Lambda \equiv 0$. For this case, the Casimir family reduces to

$$
\begin{aligned}
C(\omega, A, f) & =\int \mathrm{d}^{2} x\left(\omega-\hat{z} \times \nabla \cdot \int \mathrm{d}^{3} v f \mathbf{v}_{\perp}\right) \Phi(A) \\
& =\int \mathrm{d}^{2} x \Phi^{\prime}(A)\left(\nabla_{\perp} \psi \cdot \nabla_{\perp} A+\int \mathrm{d}^{3} v f \mathbf{v}_{\perp} \cdot \hat{z} \times \nabla A\right) \\
& =\int \mathrm{d}^{2} x \Phi^{\prime}(A)(\boldsymbol{U}-\mathbf{K}) \cdot \mathbf{B}
\end{aligned}
$$

where we recall that $\mathbf{K}=\int \mathrm{d}^{3} v f \mathbf{v}_{\perp}$, which corresponds to the momentum of the hot particle species in the $x y$-plane. Equation [14.9] introduces a hybrid cross-helicity density $(\boldsymbol{U}-\mathbf{K}) \cdot \mathbf{B}$, expressing the correlation between the magnetic field and a velocity field obtained by subtracting from the bulk velocity a contribution coming from the kinetic species. Upon setting $\mathbf{K} \equiv 0$, this Casimir reduces to the cross helicity family of invariants for two-dimensional MHD which to our knowledge was first found in [MOR 84]. For a given constant $A_{0}$, choosing $\Phi(A)=\mathscr{H}_{A_{0}}(A)$, with $\mathscr{H}$ indicating the Heaviside function with step located at $A=A_{0}$, expresses the property that not only is the total generalized cross-helicity is conserved, but also its integral over domains $A=A_{0}$, which are bounded by magnetic flux surfaces.

Given that these families of Casimirs have been identified explicitly, we are ready to apply the energy-Casimir method and find sufficient conditions for energy stability of the system. This is carried out in the next section.

\subsection{Energy-Casimir stability analysis}

Knowledge of the Casimir invariants provides a variational principle for equilibria, $\delta \mathscr{F}=0$, which we tend to in section 14.4.1. Then, the next step of the energy-Casimir method is to consider the second variation, $\delta^{2} \mathscr{F}=0$, which is done in section 14.4.2. Note that all physical constants have been set to unity. A more perspicuous study of the dynamical behavior can be obtained upon restoring these constants. 


\subsubsection{Equilibrium variational principle}

To construct a variational principle for the equilibria under consideration, we first define the free energy functional $\mathscr{F}=H+C$. Then, we take its first variation, which reads

$$
\delta \mathscr{F}=\delta \mathscr{F}_{M H D}+\delta \mathscr{F}_{V}-\delta \iint \mathrm{d}^{2} x \mathrm{~d}^{3} v \Phi(A) \mathbf{v} \cdot \hat{z} \times \nabla f
$$

where we split-off the Vlasov part $\delta \mathscr{F}_{V}:=\int \mathrm{d}^{2} x \mathrm{~d}^{3} v\left(\Lambda^{\prime}+|\mathbf{v}|^{2} / 2\right) \delta f$ and the MHD part

$$
\delta \mathscr{F}_{M H D}:=\int \mathrm{d}^{2} x\left(\Phi(A)-\Delta^{-1} \omega\right) \delta \omega+\int \mathrm{d}^{2} x\left(\omega \Phi^{\prime}(A)-\Delta A+\Psi^{\prime}(A)\right) \delta A .
$$

Upon setting $\delta \mathscr{F}=0$, the equilibrium equations turn out to be

$$
\begin{aligned}
0 & =\psi_{e}+\Phi\left(A_{e}\right), \\
0 & =-\Delta A_{e}+\bar{\omega}_{e} \Phi^{\prime}\left(A_{e}\right)+\Psi^{\prime}\left(A_{e}\right), \\
0 & =\Phi^{\prime}\left(A_{e}\right) \mathbf{v} \cdot \hat{z} \times \nabla A_{e}+\frac{|\mathbf{v}|^{2}}{2}+\Lambda^{\prime}\left(f_{e}\right),
\end{aligned}
$$

whose first relation renders the third in the form

$$
\frac{1}{2}\left|\mathbf{v}+\boldsymbol{U}_{e}\right|^{2}-\frac{1}{2}\left|\boldsymbol{U}_{e}\right|^{2}+\Lambda^{\prime}=0 .
$$

In the above expressions, we introduced the subscript $e$ to indicate equilibrium quantities.

Upon assuming an invertible $\Lambda$, from [14.13], we obtain the equilibrium distribution function in the form

$$
f_{e}=f_{e}\left(\frac{1}{2}\left|\mathbf{v}+\boldsymbol{U}_{e}\right|^{2}-\frac{1}{2}\left|\boldsymbol{U}_{e}\right|^{2}\right)=f_{e}\left(\frac{1}{2}|\mathbf{v}+\hat{z} \times \nabla \Phi|^{2}-\frac{1}{2}\left|\nabla_{\perp} \Phi\right|^{2}\right) .
$$

As an example, consider the relative Gaussian distribution

$$
f_{e}=\exp \left(-\frac{1}{2}\left|\mathbf{v}+\boldsymbol{U}_{e}\right|^{2}+\frac{1}{2}\left|\boldsymbol{U}_{e}\right|^{2}\right)
$$


It is easy to see that this yields

$$
\int \mathrm{d}^{3} v f_{e} \mathbf{v}=-e^{-\frac{1}{2}\left|\boldsymbol{U}_{e}\right|^{2}} \boldsymbol{U}_{e} \Longrightarrow \overline{\boldsymbol{\omega}}=\nabla_{\perp} \cdot\left(\left(1+e^{-\frac{1}{2}\left|\nabla_{\perp} \Phi\right|^{2}}\right) \nabla_{\perp} \Phi\right)
$$

and therefore, in the absence of MHD equilibrium flow, we have $\bar{\omega}_{e}=0$, which means that the vorticity $\hat{z} \times \nabla \cdot \int d^{3} v f \mathbf{v}_{\perp}$ associated with the hot particle flow is also zero. In the general case of an arbitrary equilibrium of the type [14.14], this quantity is computed as

$$
\begin{aligned}
\hat{z} \times \nabla \cdot \int \mathrm{d}^{3} v f_{e} \mathbf{v}_{\perp} & =\int d^{3} v f_{e}^{\prime}\left(\mathbf{v}_{\perp}+\boldsymbol{U}\right) \cdot \hat{z} \times \nabla\left[\frac{1}{2}\left|\mathbf{v}_{\perp}+\boldsymbol{U}\right|^{2}-\frac{1}{2}|\boldsymbol{U}|^{2}+\frac{1}{2} v_{z}^{2}\right] \\
& =-\nabla_{\perp} \cdot\left(n_{e} \nabla_{\perp} \Phi\right),
\end{aligned}
$$

where $n_{e}=\int d^{3} v f_{e}$ is the hot particle equilibrium density. In conclusion, the final form of the hybrid equilibrium relation reads

$$
-\Delta A_{e}+\Phi^{\prime}\left(A_{e}\right) \nabla_{\perp} \cdot\left(\left(1+n_{e}\right) \nabla_{\perp} \Phi\left(A_{e}\right)\right)+\Psi^{\prime}\left(A_{e}\right)=0
$$

In the absence of a hot species $\left(n_{e} \equiv 0\right)$, this reduces to the celebrated Grad-Shafranov equation for reduced MHD [HAZ 84]. Note, when $n_{e} \not \equiv 0$, we call the above equilibrium relation the hybrid Grad-Shafranov equation.

\subsubsection{Stability conditions}

Turning now to stability criteria, we compute the second variation

$$
\begin{gathered}
\delta^{2} \mathscr{F}=\delta^{2} \mathscr{F}_{M H D}+\delta^{2} \mathscr{F}_{V}-2 \iint \mathrm{d}^{2} x \mathrm{~d}^{3} v \delta \Phi(A) \mathbf{v}_{\perp} \cdot \hat{z} \times \nabla \delta f \\
-\iint \mathrm{d}^{2} x \mathrm{~d}^{3} v\left(\mathbf{v}_{\perp} \cdot \hat{z} \times \nabla f\right) \Phi^{\prime}(A)(\delta A)^{2}
\end{gathered}
$$

where we have introduced

$$
\begin{gathered}
\delta^{2} \mathscr{F}_{M H D}=\int \mathrm{d}^{2} x\left(|\nabla \delta \psi-\nabla \delta \Phi|^{2}+\left(1-\left(\Phi^{\prime}\right)^{2}\right)|\nabla \delta A|^{2}\right) \\
+\int \mathrm{d}^{2} x\left(\omega \Phi^{\prime \prime}+\Psi^{\prime \prime}+\Phi^{\prime} \Delta \Phi^{\prime}\right)(\delta A)^{2}, \\
\delta^{2} \mathscr{F}_{V}=\int \mathrm{d}^{2} x \mathrm{~d}^{3} v \Lambda^{\prime \prime}(f)(\delta f)^{2},
\end{gathered}
$$

which correspond to the second variation expressions for reduced MHD [HAZ 84] and the Vlasov equation [FOW 63, GAR 63, MOR 87, HOL 85, MOR 90], respectively. 
After some rearrangement, the expression for the second variation, evaluated at an equilibrium solution of [14.10]-[14.12], can be written as follows

$$
\begin{aligned}
& \delta^{2} \mathscr{F}\left(\omega_{e}, A_{e}, f_{e}\right)=\delta^{2} \mathscr{F}_{M H D}\left(\omega_{e}, A_{e}, f_{e}\right)+\delta^{2} \mathscr{F}_{V}\left(\omega_{e}, A_{e}, f_{e}\right) \\
&-\iint \mathrm{d}^{2} x \mathrm{~d}^{3} v\left(\mathbf{v} \cdot \hat{z} \times \nabla f_{e}\right) \Phi^{\prime \prime}\left(A_{e}\right)(\delta A)^{2} \\
&+\iint \mathrm{d}^{2} x \mathrm{~d}^{3} v f_{e}^{-1}\left|\delta f-\delta A f_{e} \nabla_{\perp} \Phi^{\prime}\left(A_{e}\right) \cdot \hat{z} \times \mathbf{v}_{\perp}\right|^{2} \\
&+\iint \mathrm{d}^{2} x \mathrm{~d}^{3} v f_{e}^{-1}\left|\delta f-f_{e} \Phi^{\prime}\left(A_{e}\right) \nabla_{\perp} \delta A \cdot \hat{z} \times \mathbf{v}_{\perp}\right|^{2} \\
&-2 \iint \mathrm{d}^{2} x \mathrm{~d}^{3} v f_{e}^{-1}(\delta f)^{2}-\int \mathrm{d}^{2} x\left(\operatorname{Tr} \mathbb{P}_{\perp e}\right)\left|\nabla_{\perp} \Phi^{\prime}\left(A_{e}\right)\right|^{2}(\delta A)^{2} \\
&+\iint \mathrm{d}^{2} x \mathrm{~d}^{3} v f_{e}\left(\mathbf{v}_{\perp} \cdot \nabla_{\perp} \Phi^{\prime}\left(A_{e}\right)\right)^{2}(\delta A)^{2} \\
&-\int \mathrm{d}^{2} x \Phi^{\prime 2}\left(A_{e}\right)\left(\operatorname{Tr} \mathbb{P}_{\perp e}\right)\left|\nabla_{\perp} \delta A\right|^{2} \\
&+\iint \mathrm{d}^{2} x \mathrm{~d}^{3} v f_{e} \Phi^{\prime 2}\left(A_{e}\right)\left(\mathbf{v}_{\perp} \cdot \nabla_{\perp} \delta A\right)^{2}
\end{aligned}
$$

where we have defined $\mathbb{P}_{\perp e}:=\int d^{3} v f_{e} \mathbf{v}_{\perp} \mathbf{v}_{\perp}$, while Tr denotes the ordinary matrix trace.

Because energy stability is attained (by definition) if the second variation, evaluated at the equilibrium, has a definite sign, for any perturbations $\delta A, \delta \omega$ and $\delta f$, we infer from [14.15], that sufficient conditions for stability are provided by

$$
\begin{aligned}
& \left|\Phi^{\prime}\left(A_{e}\right)\right|^{2}<\frac{1}{1+\operatorname{Tr} \mathbb{P}_{\perp e}} \\
& \bar{\omega}_{e} \Phi^{\prime \prime}\left(A_{e}\right)+\Psi^{\prime \prime}\left(A_{e}\right)+\Phi^{\prime}\left(A_{e}\right) \Delta \Phi^{\prime}\left(A_{e}\right)-\left|\nabla_{\perp} \Phi^{\prime}\left(A_{e}\right)\right|^{2} \operatorname{Tr} \mathbb{P}_{\perp e}>0 \\
& \Lambda^{\prime \prime}\left(f_{e}\right)>2 / f_{e} .
\end{aligned}
$$

Note that $\psi_{e}=-\Phi\left(A_{e}\right)$ implies $\boldsymbol{U}_{e}=-\Phi^{\prime}\left(A_{e}\right) \mathbf{B}_{e}$, so that the stability condition of [14.16] reads

$$
B_{e}>\left(1+\operatorname{Tr} \mathbb{P}_{\perp e}\right) U_{e},
$$

where $B_{e}:=\left|\mathbf{B}_{e}\right|$ and $U_{e}:=\left|\boldsymbol{U}_{e}\right|$. Because of the presence of the kinetic component, our stability condition requires slower equilibrium flows, in comparison to the corresponding condition for reduced MHD. The latter condition [MOR 84], indeed, 
requires MHD flows to be just sub-Alfvénic, whereas this is no longer sufficient to satisfy [14.16] in the presence of a hot particle population.

Upon making use of $\Phi^{\prime}=-\boldsymbol{U}_{e} \cdot \mathbf{B}_{e} / B_{e}^{2}=-U_{e} / B_{e}$, the condition [14.17] can be reformulated in the following way:

$$
\begin{array}{r}
\frac{\mathbf{B}_{e} \times \hat{z} \cdot \nabla_{\perp} J_{e}}{B_{e}^{2}}\left(1-\frac{U_{e}^{2}}{B_{e}^{2}}\right)-\frac{1}{2} \frac{\mathbf{B}_{e} \times \hat{z}}{B_{e}^{2}} \cdot \nabla_{\perp}\left(\frac{U_{e}^{2}}{B_{e}^{2}}\right) \frac{\mathbf{B}_{e} \times \hat{z}}{B_{e}^{2}} \cdot \nabla_{\perp} B_{e}^{2} \\
+\frac{U_{e}}{B_{e}} \frac{\mathbf{B}_{e} \times \hat{z}}{B_{e}^{2}} \cdot \nabla_{\perp} \hat{z} \times \nabla \cdot \mathbf{K}_{e}-\left|\nabla_{\perp}\left(\frac{U_{e}}{B_{e}}\right)\right|^{2} \operatorname{Tr} \mathbb{P}_{\perp e}>0,
\end{array}
$$

which provides an interpretation of this stability condition in terms of physical properties of the equilibrium state. The terms of line [14.19] correspond to the same terms appearing in the energy stability condition for reduced MHD. In particular, we can recognize in the first term the above-mentioned sub-Alfvénic condition, in addition to conditions depending on the relative direction of the equilibrium magnetic fields and the gradient of the current density. These have been shown to be a source for the kink and interchange instabilities observed in tokamaks in the presence of magnetic curvature, see Chapter 12. The terms of line [14.20] account for the new contributions due to the kinetic species. These are due to the compressibility of the hot particle equilibrium flow and to the hot particle energy. We observe that, in the case of static MHD equilibrium (i.e. $\boldsymbol{U}_{e}=0$ ), the presence of the kinetic species has no influence on the condition [14.19]-[14.20]. In particular, in that limit, we recover the pure reduced MHD condition [HAZ 84], $\mathbf{B}_{e} \times \hat{z} \cdot \nabla_{\perp} J_{e}>0$, which corresponds to a current density profile monotonically decreasing with the equilibrium flux function $A_{e}$.

Finally, upon differentiating the equilibrium relation for $\Lambda^{\prime}$ with respect to $\mathbf{v}$, we obtain

$$
\Lambda^{\prime \prime}\left(f_{e}\right)=-\frac{1}{f_{e}^{\prime}} \Longrightarrow f_{e}<-2 f_{e}^{\prime}
$$

For example, the particular Gaussian distributions such that $f_{e}^{\prime}=-f_{e}$ (unit variance) are stable equilibria. This is a modification of Gardner's well-known monotonicity theorem [GAR 63, FOW 63].

It should be emphasized that our sufficient conditions are not optimal. Clearly, some potentially stabilizing positive definite terms have not been used, and terms involving gradients, e.g., $\left|\nabla_{\perp} \delta A\right|^{2}$, could be estimated in conjunction with those involving $(\delta A)^{2}$ by the Poincaré inequality in order to obtain better results. Also, the conditions we have obtained control $\delta A$ and $\delta f$, but an examination of $\delta^{2} \mathscr{F}_{M H D}$ 
reveals that there is a neutral direction given by $\delta \psi=\delta \Phi$. This was pointed out in [MOR 86], where it was shown that this corresponds to the Alfvén wave that has an interpretation in terms of spontaneous symmetry breaking and plays the role of the Goldstone mode of particle physics.

\subsection{Conclusions}

Stability analyses play a central role in the investigation of phenomena occurring in plasmas. For phenomena in which dissipative effects can be neglected, plasma models should be energy conserving and possess a Hamiltonian structure, thereby avoiding unphysical "phantom dissipation". For continuum models (e.g. kinetic or fluid theories) formulated in terms of Eulerian variables, the Hamiltonian structure is generically of noncanonical type. Associated with such Hamiltonian structure are Casimir functionals, particular invariants arising from degeneracy of the cosymplectic (bivector) operator. The existence of Casimir invariants is the basis of the energy-Casimir method for determining stability conditions for noncanonical Hamiltonian systems, which, as mentioned in section 14.2, imply spectral stability. In this chapter, we applied the energy-Casimir method to the planar version of a hybrid model for plasmas, which couples, via the pressure terms, the dynamics of a bulk MHD flow, with the kinetic evolution of a population of hot particles.

After introducing the model, we formulated its Hamiltonian structure, pointing out how it relates to the Hamiltonian structures of reduced MHD and the Vlasov equation. In particular, in terms of the adapted variables, it emerged that the corresponding noncanonical Poisson bracket introduces the kinetic-MHD coupling terms, whereas the Hamiltonian is just the sum of the reduced MHD and Vlasov contributions. This was also reflected in the Casimir structure. Indeed, the latter was seen to be divided into three independent contributions: two of these inherited from reduced MHD and Vlasov equation, which correspond to the magnetic frozen-in condition and to the conservation of any function of $f$ integrated over phase space, respectively. However, the third family of Casimirs that was seen to originate from the coupling terms in the bracket is peculiar to this model and expresses the conservation of a generalized hybrid cross-helicity, which, unlike the usual cross-helicity of MHD, accounts also for the contribution of the fluid momentum of the hot particle species.

The abundance of Casimirs present in this planar reduction of the model facilitated the application of the energy-Casimir method. From the first variation of the free energy functional $\mathscr{F}$, we determined general equations for equilibria of the system. These led, in particular, to a hybrid Grad-Shafranov equation, which generalizes the traditional equilibrium conditions of two-dimensional MHD. Finally, explicit energy stability conditions were obtained from the analysis of the second 
variation of $\mathscr{F}$. On the basis of the obtained conditions, the presence of the hot particle species was seen to impose a lower bound on the equilibrium bulk speed when compared to the pure MHD case. The presence of the kinetic component was also seen to require stronger conditions on the current density profile for the stability to be attained. The distribution function, on the other hand, is constrained by dependence on the MHD component, via the equilibrium relation, and its variation in terms of the equilibrium quantities required a bound from above in order to satisfy the stability conditions. However, also in the presence of the hot particle population, we observed that without MHD equilibrium flow, a monotonically decreasing current density profile satisfies the stability condition, as is the case for reduced MHD. Also, Gaussian distribution functions with unit variance are seen to satisfy the equilibrium condition, as is the case for purely kinetic Vlasov-like systems.

In conclusion, we remark that the conditions obtained are not optimal; further analysis of the functional $\mathscr{F}$ could lead to tighter conditions. However, the energyCasimir analysis as performed is direct and efficient and circumvents more detailed spectral analysis.

\subsection{Acknowledgments}

P.J. Morrison was supported by US Department of Energy, grant no. DE-FG02-04ER54742. This work was supported by the European Community under the contracts of Association between EURATOM, CEA and the French Research Federation for fusion studies. The views and opinions expressed herein do not necessarily reflect those of the European Commission. E. Tassi received financial support from the Agence Nationale de la Recherche (ANR GYPSI) and from the CNRS (PEPS project GEOPLASMA).

\subsection{Appendix A: derivation of hybrid Hamiltonian structure}

In this appendix, we obtain the Hamiltonian structure composed of the Hamiltonian of [14.6] and Poisson bracket of [14.7], by restriction of the Hamiltonian structure first given in [TRO 10] for the full three-dimensional HPCS model. (For background material see, e.g., [MOR 98, MOR 05] and [AND 12] for a similar derivation). 
The HPCS Poisson bracket is given by

$$
\begin{aligned}
\{F, G\}= & \int \mathrm{d}^{3} x \mathbf{M} \cdot\left(\frac{\delta G}{\delta \mathbf{M}} \cdot \nabla \frac{\delta F}{\delta \mathbf{M}}-\frac{\delta F}{\delta \mathbf{M}} \cdot \nabla \frac{\delta G}{\delta \mathbf{M}}\right) \\
& -\int \mathrm{d}^{3} x \rho\left(\frac{\delta F}{\delta \mathbf{M}} \cdot \nabla \frac{\delta G}{\delta \rho}-\frac{\delta G}{\delta \mathbf{M}} \cdot \nabla \frac{\delta F}{\delta \rho}\right) \\
& +\int \mathrm{d}^{3} x \mathbf{B} \cdot\left(\frac{\delta F}{\delta \mathbf{M}} \times \nabla \times \frac{\delta G}{\delta \mathbf{B}}-\frac{\delta G}{\delta \mathbf{M}} \times \nabla \times \frac{\delta F}{\delta \mathbf{B}}\right) \\
& +\int \mathrm{d}^{3} x \mathrm{~d}^{3} v f\left(\left[\frac{\delta F}{\delta f}, \frac{\delta G}{\delta f}\right]_{v}+\mathbf{B} \cdot \frac{\partial}{\partial \mathbf{v}} \frac{\delta F}{\delta f} \times \frac{\partial}{\partial \mathbf{v}} \frac{\delta G}{\delta f}\right) \\
& +\int \mathrm{d}^{3} x \mathrm{~d}^{3} v f\left(\left[\frac{\delta F}{\delta f}, \mathbf{v} \cdot \frac{\delta G}{\delta \mathbf{M}}\right]_{v}-\left[\frac{\delta G}{\delta f}, \mathbf{v} \cdot \frac{\delta F}{\delta \mathbf{M}}\right]_{v}\right)
\end{aligned}
$$

whereas the Hamiltonian reads

$$
H=\int \mathrm{d}^{3} x\left(\frac{|\mathbf{M}|^{2}}{2 \rho} \mathrm{d}^{3}+\rho \mathscr{U}(\rho)+\frac{|\mathbf{B}|^{2}}{2}\right)+\frac{1}{2} \int \mathrm{d}^{3} x \mathrm{~d}^{3} v f|\mathbf{v}|^{2} .
$$

In equations [14.21) and [14.22], $\rho$ and $\mathbf{M}$ indicate the mass density of the bulk fluid and its momentum density, respectively, whereas $\mathscr{U}(\rho)$ is the internal energy per unit mass. Physical constants have been set equal to unity.

The proof that [14.22] satisfies the Jacobi identity also can be carried out by explicit verification. Indeed, upon recognizing that it is composed of terms of the original bracket of MHD [MOR 80b] and that of the Maxwell-Vlasov system [MOR 80a, MOR 82, MAR 82, MAR 83], together with later work on the two-fluid system [SPE 82a, SPE 82b], it is not difficult to ascertain the validity of the Jacobi identity. Alternatively, we can begin with an action principle (see, e.g., [MOR 09]), in particular the action principle for this model of [HOL 12], and derive the Poisson bracket of [14.22], thereby ensuring the Jacobi identity.

To see how the bracket [14.21] reduces to the bracket [14.7] of the planar model, we first consider the two-dimensional restriction of [14.21] by eliminating the dependence on the $z$ coordinate. Then, we can enforce incompressibility by restricting to functionals that are independent of $\rho$ in [14.21]. Consistently, we also remove the internal energy term from the Hamiltonian [14.22]. We note that with such restrictions on the functionals and on the Poisson bracket, the Poisson bracket loses all explicit functional dependence on $\rho$. Thus, according to the bracket theorem of [MOR 82], the restricted Poisson bracket must satisfy the Jacobi identity. 
By using vector identities, we can rewrite the first line of [14.21] as

$$
\begin{aligned}
& \int \mathrm{d}^{2} x \mathbf{M} \cdot\left(\frac{\delta G}{\delta \mathbf{M}} \cdot \nabla \frac{\delta F}{\delta \mathbf{M}}-\frac{\delta F}{\delta \mathbf{M}} \cdot \nabla \frac{\delta G}{\delta \mathbf{M}}\right) \\
& =\int \mathrm{d}^{2} x\left(\nabla \times \mathbf{M} \cdot\left(\frac{\delta F}{\delta \mathbf{M}} \times \frac{\delta G}{\delta \mathbf{M}}\right)-\frac{\delta F}{\delta \mathbf{M}} \nabla \cdot \frac{\delta G}{\delta \mathbf{M}}+\frac{\delta G}{\delta \mathbf{M}} \nabla \cdot \frac{\delta F}{\delta \mathbf{M}}\right) .
\end{aligned}
$$

Then, introduction of the relations

$$
\nabla \times \mathbf{M}=\omega \hat{z}, \quad \mathbf{B}=\nabla \times(A \hat{z})
$$

leads to the following rule for transforming the functional derivatives

$$
\frac{\delta F}{\delta \mathbf{M}}=\nabla \times\left(\frac{\delta \bar{F}}{\delta \omega} \hat{z}\right), \quad \hat{z} \cdot \nabla \times \frac{\delta F}{\delta \mathbf{B}}=\frac{\delta \bar{F}}{\delta A}
$$

Using [14.24] and [14.25], together with [14.23] in [14.21], leads namely to the bracket [14.7] of the incompressible planar model. The Hamiltonian [14.22], on the other hand, reduces to [14.6].

\subsection{Appendix B: Casimir verification}

Here, we demonstrate explicitly that [14.8] is a Casimir invariant, i.e. satisfies $\{F, C\}=0$ for all functionals $F$. This is simplified by noting that $\int \mathrm{d}^{2} x \Psi(A)$ and $\int \mathrm{d}^{2} x \mathrm{~d}^{3} v \Lambda(f)$ are separate Casimirs of [14.7]. Using $\delta C / \delta f=\mathbf{v} \cdot \hat{z} \times \nabla \Phi$, we obtain

$$
\begin{aligned}
\{F, C\}=\int \mathrm{d}^{2} x \omega & {\left[\frac{\delta F}{\delta \omega}, \Phi\right]+\int \mathrm{d}^{2} x A\left(\left[\frac{\delta F}{\delta \omega}, \bar{\omega} \Phi^{\prime}\right]-\left[\Phi, \frac{\delta F}{\delta A}\right]\right) } \\
& -\int \mathrm{d}^{2} x \mathrm{~d}^{3} v f\left(\left[\frac{\delta F}{\delta f}, \mathbf{v} \cdot \hat{z} \times \nabla \Phi\right]_{v}-\left[\mathbf{v} \cdot \hat{z} \times \nabla \Phi, \mathbf{v} \cdot \hat{z} \times \nabla \frac{\delta F}{\delta \omega}\right]_{v}\right) \\
& +\int \mathrm{d}^{2} x \mathrm{~d}^{3} v f\left[\frac{\delta F}{\delta f}, \mathbf{v} \cdot \hat{z} \times \nabla \Phi\right]_{v} \\
=\int \mathrm{d}^{2} x \omega & {\left[\frac{\delta F}{\delta \omega}, \Phi\right]-\int \mathrm{d}^{2} x \bar{\omega}\left[\frac{\delta F}{\delta \omega}, \Phi\right] } \\
& +\int \mathrm{d}^{2} x \mathrm{~d}^{3} v f\left[\mathbf{v} \cdot \hat{z} \times \nabla \Phi, \mathbf{v} \cdot \hat{z} \times \nabla \frac{\delta F}{\delta \omega}\right]_{v}
\end{aligned}
$$




$$
\begin{aligned}
=\int \mathrm{d}^{2} x \mathrm{~d}^{3} v \mathbf{v} \cdot \hat{z} \times \nabla f\left[\frac{\delta F}{\delta \omega}, \Phi\right] \\
-\int \mathrm{d}^{2} x \mathrm{~d}^{3} v f\left(\left[\mathbf{v} \cdot \hat{z} \times \nabla \Phi, \frac{\delta F}{\delta \omega}\right]-\left[\mathbf{v} \cdot \hat{z} \times \nabla \frac{\delta F}{\delta \omega}, \Phi\right]\right) \\
=\int \mathrm{d}^{2} x \mathrm{~d}^{3} v \mathbf{v} \cdot \hat{z} \times \nabla f\left[\frac{\delta F}{\delta \omega}, \Phi\right]-\int \mathrm{d}^{2} x \mathrm{~d}^{3} v f\left(\mathbf{v} \cdot \hat{z} \times \nabla\left[\Phi, \frac{\delta F}{\delta \omega}\right]\right. \\
\left.-\left[\Phi, \mathbf{v} \cdot \hat{z} \times \nabla \frac{\delta F}{\delta \omega}\right]-\left[\mathbf{v} \cdot \hat{z} \times \nabla \frac{\delta F}{\delta \omega}, \Phi\right]\right)=0 .
\end{aligned}
$$

In the above computations, integrations by parts with vanishing boundary terms have been carried out and the Leibniz identity has been used. Also, prime denotes differentiation with respect to the argument.

\subsection{Bibliography}

[AND 12] Andreussi T., Morrison P.J., Pegoraro F., "Hamiltonian magnetohydrodynamics: symmetric formulation, Casimir invariants, and equilibrium variational principles", Physics of Plasmas, vol. 19, pp. 052102-1-052102-8, 2012.

[BAT 95] BATT J., MORRISON P.J., REIN G., "Linear stability of stationary solutions of the Vlasov-Poisson system in three dimensions", Archive for Rational Mechanics and Analysis, vol. 130, pp. 163-182, 1995.

[CHE 91] Cheng C.Z., "A kinetic-magnetohydrodynamic model for low-frequency phenomena", Journal of Geophysical Research, vol. 96, no. A12, pp. 21159-21171, 1991.

[FOW 63] FOWLER K., "Lyapunov's stability criteria for plasmas", Journal of Mathematical Physics, vol. 4, pp. 559-609, 1963.

[FU 95] FU G.Y., PARK W., "Nonlinear hybrid simulation of the toroidicity-induced Alfvén Eigenmode", Physical Review Letters, vol. 74, pp. 1594-1596, 1995.

[GAR 63] GARDNER C.S., "Bound on the energy available from a plasma", Physics of Fluids, vol. 6, pp. 839-840, 1963.

[HAZ 84] Hazeltine R.D., Holm D.D., Marsden J.E., Morrison P.J., "Generalized Poisson brackets and nonlinear Lyapunov stability-application to reduced MHD", in TRAN M.Q., SAWley M.L. (eds), International Conference on Plasma Physics Proceedings, Polytechnique Federal School of Lausanne, pp. 203-211, 1984.

[HOL 85] Holm D.D., Marsden J.E., Ratiu T. S., et al., "Nonlinear stability of fluid and plasma equilibria", Physics Reports, vol. 123, pp. 1-116, 1985.

[HOL 12] Holm D.D., Tronci C., "Euler-Poincaré formulation of hybrid plasma models", Communications in Mathematical Sciences, vol. 10, pp. 191-222, 2012. 
[KIM 04] Kim C.C., SovineC C.R., PARKer S.E., "Hybrid kinetic-MHD simulations in general geometry", Computer Physics Communications, vol. 164, pp. 448-455, 2004.

[MAR 82] MARSDEN J.E., WEINSTEIN A., "The Hamiltonian structure of the MaxwellVlasov equations", Physica D, vol. 4, pp. 394-406, 1982.

[MAR 83] Marsden J.E., Weinstein A., Ratiu T.S., et al., "Hamiltonian systems with symmetry, coadjoint orbits and plasma physics", Atti della Accademia delle Scienze di Torino. Classe di Scienze Fisiche, Matematiche e Naturali, vol. 117, pp. 289-340, 1983.

[MAR 84] MARSDEN J.E., MorRison P.J., "Noncanonical Hamiltonian field theory and reduced MHD”, Contemporary Mathematics, vol. 28, pp. 133-150, 1984.

[MOR 80a] MORRISON P.J., "The Maxwell-Vlasov equations as a continuous Hamiltonian system”, Physics Letters A, vol. 80, pp. 383-386, 1980.

[MOR 80b] MORRISON P.J., GREENE J.M., "Noncanonical Hamiltonian density formulation of hydrodynamics and ideal magnetohydrodynamics", Physical Review Letters, vol. 45, pp. 790-794, 1980.

[MOR 82] MORRISON P.J., "Poisson brackets for fluids and plasmas", in TABOR M., TREVE Y. (eds), Mathematical Methods in Hydrodynamics and Integrability in Dynamical Systems, AIP Conference Proceedings, pp. 13-46, 1982.

[MOR 84] MORRISON P.J., HAZELTINE R.D., "Hamiltonian formulation of reduced magnetohydrodynamics", Physics of Fluids, vol. 27, pp. 886-897, 1984.

[MOR 86] MORRISON P.J., EliEZER S., "Spontaneous symmetry breaking and neutral stability in the noncanonical Hamiltonian formalism", Physical Review A, vol. 33, pp. 4205-4214, 1986.

[MOR 87] MORRISON P.J., "Variational principle and stability of nonmonotonic VlasovPoisson equilibria", Zeitschrift für Naturforschung, vol. 42a, pp. 1115-1123, 1987.

[MOR 90] MORrison P.J., Kotschenreuther M., "The free energy principle, negative energy modes and stability”, in BAR'YAKHTAR V.G., CHERNOUSENKO V.M., EROKHIN N.S., SitenKo A.B., ZaKharov V.E. (eds), Nonlinear World. IV International Workshop on Nonlinear and Turbulent Processes in Physics, Singapore, World Scientific, pp. 910932, 1990.

[MOR 98] MORRISON P.J., "Hamiltonian description of the ideal fluid", Reviews of Modern Physics, vol. 70, pp. 467-521, 1998.

[MOR 05] MORRISON P.J., "Hamiltonian and action principle formulations of plasma physics", Physics of Plasmas, vol. 12, pp. 058102-1-058102-13, 2005.

[MOR 09] MORRISON P.J., "On Hamiltonian and action principle formulations of plasma dynamics", in Eliasson B., SHUKLA P. (eds), New Developments in Nonlinear Plasma Physics: Proceedings for the 2009 ICTP College on Plasma Physics, AIP Conference Proceedings, La Jolla, pp. 329-344, 2009.

[MOR 13] MORRISON P.J., TASSI E., TRONKO N., "Stability of compressible reduced magnetohydrodynamic equilibria-analogy with magnetorotational instability", Physics of Plasmas, vol. 20, pp. 042109-1-042109-10, 2013. 
[PAR 92] PARK W., et al., "Three-dimensional hybrid gyrokinetic-magnetohydrodynamics simulation", Physics of Fluids B, vol. 4, pp. 2033-2037, 1992.

[PAR 99] PARK W., Belova E.V., Fu G.Y., et al., "Plasma simulation studies using multilevel physics models", Physics of Plasmas, vol. 6, pp. 1796-1803, 1999.

[REI 94] REIN G., "Nonlinear stability for the Vlasov-Poisson system - the energy-Casimir method", Mathematical Methods in the Applied Sciences, vol. 17, pp. 1129-1140, 1994.

[SPE 82a] SPENCER R.G., "The Hamiltonian structure of multi-species fluid electrodynamics", in TABOR M., TREVE Y. (eds), Mathematical Methods in Hydrodynamics and Integrability in Dynamical Systems, AIP Conference Proceedings, La Jolla, California, pp. 121-126, 1982.

[SPE 82b] SPENCER R.G., KAUfMAN A.N., "Hamiltonian structure of two-fluid plasma dynamics”, Physical Review A, vol. 25, pp. 2437-2439, 1982.

[TAK 09] TAKAHASH R., BRENNAN D.P., Kim C.C., "Kinetic effects of energetic particles on resistive MHD stability", Physical Review Letters, vol. 102, pp. 135001-1-135001-4, 2009.

[TRO 10] TRONCI C., "Hamiltonian approach to hybrid plasma models", Journal of Physics A: Mathematical and Theoretical, vol. 43, pp. 375501-1-375501-26, 2010.

[TRO 13] Tronci C., TASSI E., MorRison P.J., "Hybrid Vlasov-MHD models: Hamiltonian vs. non-Hamiltonian”, arXiv, 1306.2406, 2013.

[YOS 13] Yoshida Z., Morrison P. J., Dobarro F., "Singular Casimir elements of the Euler equation and equilibrium points", Journal of Mathematical Fluid Mechanics, 2013. 